\title{
A dietary supplement improves age-related dermatological changes. Observational, anecdotal, spontaneous study
}

\author{
Carmen Laurino ${ }^{1 *}$, Beniamino Palmieri², Alessandro Coacci ${ }^{3}$, Maria Vadalà ${ }^{1}$ \\ ${ }^{1}$ Research Associate, ${ }^{2}$ Associate Professor, Department of General Surgery and Surgical Specialities, University of \\ Modena and Reggio Emilia, Network of the Second Opinion, Modena, Italy, ${ }^{3}$ Medical Doctor, Department of Urgency \\ Surgery and First Aid, USL Grosseto, Italy
}

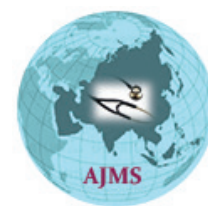

A B S T R A C T

Background: The rate of people with significant dermis and epidermis disorders increases linearly with age. The majority of skin changes are a consequence of cumulative exposure to environmental factors, such as chronic ultraviolet exposure. Aims and Objectives: Age-related dermatological changes include skin laxity, nails fragility, hair loss and hair breakage. This spontaneous, anecdotal observational study investigates if a dietary supplementation based on collagen, chondroitin sulfate, low-molecular weight hyaluronic acid, Pycnogenol ${ }^{\circledR}$ and coenzyme $\mathrm{Q} 10$ can improve age-related dermatological changes. Materials and Methods: Thirty four patients received the dietary supplement for 2 times/daily for 12 weeks. Facial skin hydration, Trans Epidermal Water Loss (TEWL) and elasticity were assessed at baseline and after 12 weeks in five selected areas. Nails hardness of the index, middle and ring fingers were also assessed at baseline and at followup by a durometer and by a nail score. The Hair Mass Index (HMI) and the Hair Breakage Index (HBI) were assessed by a mechanical device together with hair score at baseline and after 12 weeks. Results: After 12 weeks skin hydration, TEWL and elasticity significantly improved in all the tested areas $(p<0.01$ and $p<0.001)$. Nails hardness of index, middle and ring finger significantly increased $(p<0.001)$ after 12 weeks. HMI and HBI were both significantly reduced ( $p<0.0001$ ) after 12 weeks. In addition, both nails and hair score improved. Conclusions: Dietary supplementation significantly improved age-related dermatological changes.

Key words: Skin physiology/structure, Hair treatment, Formulation skin care/stability/ texture/emulsions, Ageing, Nails structure

\section{INTRODUCTION}

Skin ageing involves also its annexes, such as hair and nails. Hair follicles are produced during embryogenesis. ${ }^{1}$ In the postnatal life, hair follicle grows through three distinct phases: (i) anagen (growth) phase; (ii) catagen (regressing) phase; and (iii) telogen (resting) phase. ${ }^{2}$ This puzzling selfrenewal property is maintained by the stem cell niche located in the bulge portion of the hair follicle. ${ }^{3}$ The hair follicle has a highly complex structure with eight distinct cell layers. The nail is hard keratin adnexa, produced by the same germinative epithelium and process through which epidermal skin cells produce the stratum corneum. Nail keratinocytes contribute to cornification through the production of hard keratins and the hardness of the nail plate is due to a high concentration of sulfur matrix protein. $^{4}$

The rate of people with significant dermis and epidermis disorders increases linearly with age: at 70 years about $70 \%$ of the population claims at least one related-skin problem. ${ }^{5}$ The loss of hydration, appearance of fine wrinkles, roughness, dryness, thickening, laxity, telangiectasia, and loss of tensile strength are common features of the 
aged skin. ${ }^{6}$ Aged skin and annexes are structurally and functionally different compared to that of younger people: the epidermis is thinner and it is characterized by a slower cell turnover rate with a consequent lesser resistance to external injuries. The hydrophilia of the stratum corneum is reduced together with eccrine and sebaceous glands activity, causing skin dryness and pruritus. ${ }^{7}$ Furthermore, modification of dermal collagen and elastin promotes skin wrinkles. Histologically, the dermis is thinner, collagen fibers are fragmented and the content of elastic fibers, fibroblasts, glycosaminoglycans (GAGs) and proteoglycans (PGs) is reduced. A disappearance of microvilli in dermis-epidermis junction with defective adhesion of the epidermis to the dermis is also remarkable. ${ }^{8}$ Hair becomes gray and rarefied and nails growth is slower. ${ }^{9}$ The majority of skin changes are a consequence of cumulative exposure to environmental factors, such as chronic ultraviolet (UV) exposure. ${ }^{10} \mathrm{UV}$ radiation causes mutations by generating Reactive Oxygen Species (ROS), such as superoxide anion, hydrogen peroxide and hydroxyl radical. ${ }^{11}$ Similarly to the skin, hairs are exposed to noxious environmental factors, including UV radiation and smoking that contribute in bald scalp. ${ }^{12}$ In addition, the quantity and the quality of hair and nails are closely related to the individual nutritional state and diet. A sufficient and balanced uptake and absorption of proteins, trace elements and vitamins are fundamental in the turnover of tissues characterized by a high biosynthetic activity, such as skin and its annexes. In case of low protein intake and malnutrition with low uptake of essential aminoacids, trace element and vitamin imbalances, hair growth and pigmentation is usually impaired..$^{13,14}$

In recent years, the dietary supplements use is increasing both in Europe and in the USA: ${ }^{15}$ the bioactive compounds present in dietary supplements can be absorbed and modulate the physiological processes better than the same compounds absorbed with daily diet that can be partially or totally inactivated ${ }^{16}$ by the cooking processes. The oral administration of nutraceuticals and cosmeceuticals has the advantage to directly release bioactive compounds in blood stream and then to the targeted organs, and tissues enclosed skin, hair and nails. ${ }^{14}$ In 2007 Jacquet et al. ${ }^{16}$ reported an high efficacy of a dietary supplement containing $100 \mathrm{mg}$ shark cartilage, $1.6 \mathrm{mg}$ vitamin $\mathrm{B} 2,6 \mathrm{mg}$ vitamin $\mathrm{B} 5,2 \mathrm{mg}$ vitamin $\mathrm{B}$, $0.150 \mathrm{mg}$ vitamin $\mathrm{B} 8$, and $350 \mathrm{mg}$ fish oil on skin, hairs, and nails in two open clinical trials (total of 52 women). The dietary supplementation lasted 58 days and at the end of the treatment skin hydration, wrinkle depth and volume, hair loss and nail conditions significantly improved. The efficacy of several minerals (i.e. zinc, selenium, iron), ${ }^{16-20} \mathrm{~B}$-vitamins, ${ }^{16,21,22} \mathrm{~L}$-cystine, ${ }^{16,21,23}$ essential fatty acids, ${ }^{16}$ green tea ${ }^{16}$ and grape extract ${ }^{16}$ was also proved on hairs and nails.
We have previously verified that a dietary supplement containing collagen, chondroitin sulfate, low molecular weight hyaluronic acid (HA), Pycnogenol ${ }^{\circledR}$ and coenzyme Q10 significantly improved VAS photoaging score, sebum, hydration and tonicity of the skin, together with the increase of serum fibronectin and HA concentrations and a decrease of serum neutrophil elastase 2 and carbonylated protein concentrations starting from the second week of treatment. ${ }^{24}$ The object of this second study is to confirm that this supplement affects several skin face parameters [i.e. hydration, trans epidermal water loss (TEWL) and elasticity] and to confirm the hypothesis that it can also improves nails hardness, hair loss and hair breakage in patients affected by age-related dermatological changes.

\section{MATERIALS AND METHODS}

This study was performed through the Network of the Second Opinion (Modena, Italy), on a group of patients spontaneously asking for specific solutions available to follow a specific supplementation daily treatment. The investigation method was in accordance with the Declaration of Helsinki and approved by the local Institutional Review Board. The patients signed the informed consent before starting the dietary supplementation protocol.

\section{Patients}

Thirty-one female patients $(91.2 \%)$ and 3 male patients (8.8\%) [age: $54.1 \pm 1.8$ years (mean \pm standard error of the mean [SEM])], affected by moderate $(40 \mathrm{~mm}<$ Visual Analogue Scale (VAS) $<70 \mathrm{~mm} ; \mathrm{n}=17$ ) and severe (VAS $>70 \mathrm{~mm} ; \mathrm{n}=17)$ facial photoageing, nails fragility and rarefaction of hair follicles, were enrolled. Patients were instructed to shallow a dietary supplement, VISCODERM ${ }^{\circledR}$ Pearls (distributed by IBSA FARMACEUTICI ITALIA Srl, Lodi, Italy). All patients show a Fitzpatrick skin type 2 or 3. Inclusion criteria were moderate, severe facial photoaging as determined by VAS at baseline, nails fragility, modifications of nail layer color and hair follicle rarefaction as determined by clinic examination at baseline. Exclusion criteria were: skin allergies, face dermatitis, pre-cancerous facial skin lesions due to prolonged UV exposure and skin or general symptoms of intolerance/allergy to the components of dietary supplement evaluated in this study.

General information about patients, like drug assumption, smoke habits and diet were also collected at baseline. All these parameters influence age-related dermatologic changes. ${ }^{16}$

Patients declared if they were right or left-handed and the length [expressed in centimeters $(\mathrm{cm})$ ] of the hairs. Indeed, nail parameter clinical evaluation was done on the right 
hand for right-handed patients and on the left hand for lefthanded patients. The length of hairs was also considered a parameter for hair clinical evaluation. Smokers were classified according the number of daily smoked cigarettes (less than 5 cigarettes, between 5 and 10 cigarettes, and more than 10 cigarettes). Hair length was classified as follow: less than $3 \mathrm{~cm}$, between 3 and $11 \mathrm{~cm}$, and more than $11 \mathrm{~cm}$. In addition, women declared if they were in menopause or non-menopause state, and if they take contraception or hormone replacement therapy, since hormonal status influence age-related dermatologic changes. ${ }^{25}$

\section{Supplementation Treatment}

Patients received VISCODERM ${ }^{\circledR}$ Pearls containing collagen (124 mg), chondroitin sulfate $(40 \mathrm{mg})$, glucosamine sulfate (not less than 3\%), low-molecular-weight HA, (20 mg), Pycnogenol $^{\circledR}(15 \mathrm{mg})$, and coenzyme Q10 (10 mg) two times a day for 12 weeks The composition of VISCODERM ${ }^{\circledR}$ Pearls has been already discussed in our previous study. ${ }^{24}$ Patients received a diet based on individual's needs (including 20\% proteins, $50 \%$ carbohydrates and 30\% fats) in order to allow a comparison within the group without any diet-related bias.

\section{Skin Parameters Clinical Evaluation}

A DermaLab ${ }^{\circledR}$ USB Single Parameter Units [manufactured and distributed by Cortex Technologies (Hadsund, Denmark)] was used to quantify facial skin hydration and TEWL at baseline and after 12 weeks (follow-up). An indentation instrument for skin dermis elasticity measurement [ElastiMeter ${ }^{\circledR}$ manufactured by Delfin Technologies (Kuopio, Finland) and distributed by ForLab Italia (Bergamo, Italy)] was used to quantify facial skin elasticity.

Hydration was expressed by conductance principle in microSiemens units $(\mu S)$, TEWL was measured by diffusion gradient in $\mathrm{g} / \mathrm{m}^{2} / \mathrm{h}$, and elasticity was measured as stiffness in $\mathrm{N} / \mathrm{m}$. Stiffness may be defined as a physical measure of an object's resistance to change in shape under an external force.

The DermaLab® USB with hydration probe and module relies upon the conductance measurement principle, which is known to measure the water binding capacity of the stratum corneum. ${ }^{26}$ The instrument has a pin probe featuring eight pin electrodes that is pressed upon the skin. The DermaLab ${ }^{\circledR}$ USB with TEWL displays tip probe sensors pressed upon the skin. Sensors are protected to rule out bias due to atmospheric variations and current and direct incident light, gaps usually faced with the open chamber method. ${ }^{26}$ The value of hydration and TEWL is the arithmetic mean of three repetitive measures in the same area.

One of the most vital functions of the skin is to protect the underlying tissues both against dehydration and against environmental risk such as UV exposure, overheating or hypothermia, mechanical frictions, and chemical irritants. ${ }^{27}$ The quality of this barrier function is typically assessed by measuring TEWL, is defined as a measurement of the quantity of water that passes from inside a body through the epidermal layer to the surrounding atmosphere via diffusion and evaporation processes, which is physiologically reduced during age. ${ }^{28}$

ElastiMeter ${ }^{\circledR}$ consists of a $1 \mathrm{~mm}$ length indenter, a reference plate and built-in force sensors. ${ }^{29}$ The probe head is quickly pressed against the skin surface with a recommended standard pressure that is displayed on the screen. The indenter induces a constant deformation when the reference plate is in full contact with the skin. The skin resists the deformation and its instant elasticity is determined by ElastiMeter ${ }^{\mathbb{B}}$. Skin elasticity value is assessed as the result of the mean of five repetitive measurements assessed in the same area.

All the parameters were measured on five selected areas: forehead (F), right and left malar area (RMA and LMA, respectively), and right and left lateral-medial area of the neck ( $\mathrm{RN}$ and $\mathrm{LN}$, respectively). We assessed also the elasticity of the forearm (FO) as control, according to the manufacturer's instructions, since skin arm elasticity changes less quickly then skin face elasticity.

Patients were instructed to not use cosmetics, cream, or any other topic lotion in face, neck and forearm the day of the clinic examination, but to not change their daily cosmetic habits.

Skin parameter clinical evaluation was assessed at baseline and after 12 weeks of treatment (follow-up).

Nails parameters clinical evaluation: A durometer device [Durometer RR15 ${ }^{\circledR}$, distributed by Rupac Srl (Milano, Italy)] was used to quantify nails hardness. The device is usually employed to assess hardness of different kind of rubber and plastics. The data reading is direct and immediate and occurs on the analogic display, by a lock amount needle, which is expressed in Shore A (hardness range: 5 - 95 Shore A). Nails hardness has been detected by this durometer as previously done in two clinical studies. ${ }^{30,31}$ Its tip is pressed a few seconds directly on the nail surface of the index (IN), the medium (ME) and the ring (RI) finger of the right or the left hand, (in right-or left-handed individuals, respectively). ${ }^{32}$ The hardness value is assessed as the mean value of three repetitive measures in the same area of each finger.

A nail score (1-4) was furtherly used to assess the nail surface appearance as follow: $1=$ yellowish and breakable; $2=$ yellowish and soft; $3=$ whitish and rough; $4=$ whitish and rigid. ${ }^{14}$

Asian Journal of Medical Sciences | May-Jun 2017 | Vol 8 | Issue 3 
Patients were instructed to not apply nail enamel during all the study period and to not cut their nails the same day of clinic examination.

Nails parameter clinical evaluation was assessed at baseline and after 12 weeks of treatment (follow-up) on nails of the right hand for right-handed patients and on nails of the left hand for left-handed patients.

\section{Hair Parameters Clinical Evaluation}

A point of care HairCheck ${ }^{\circledR}$ device [manufactured by Divi International Co. (Miami, USA) and distributed by Iberius LLC (Miami, FL)] was used to quantify hair loss and hair breakage. The device is a self-contained mechanical unit with a rectangular anodized aluminum body that is held in the right hand like a hypodermic syringe. Extending from one end of the body is a hook-shaped arm, and from the other end of the body, a spring-loaded shaft with a retainer cap. The hook and the cap are at opposite ends of one contiguous shaft. An electronic sensing unit with LED display is externally mounted on the side of the housing and attached internally to the shaft. When the cap at the end of the shaft is pressed with the thumb, the hook arm extends out of the body at the opposite end, and its travel distance is displayed on the LED screen in hundredths of a millimeter. When the thumb is released, the hook retracts back toward the body. ${ }^{33}$ This mechanical device compresses a bundle of hair in a disposable cartridge from a delineated area of the scalp and measures its cross-sectional area. The device measures Hair Mass Index (HMI) in the area of the scalp. Control HMI is also measured in a fixed area of the head according to manufacturer's instructions. HMI is the ratio of the cross-sectional area of an isolated bundle of hair and the area of skin from which it was taken and represents the $\%$ value of hair loss. It is also defined as Trichometric Index (TI): $\mathrm{mm}^{2}$ of hair bundle $/ \mathrm{cm}^{2}$ of skin $\mathrm{x} 100\left(\mathrm{~mm}^{2} /\right.$ $\mathrm{cm}^{2} \mathrm{x}$ 100). The measured density (number of hairs $/ \mathrm{cm}^{2}$ ) and diameter $(\mu \mathrm{m})$ of hairs are determinants of its quantity. Measurement locations were selected according to the manufacturer's instructions. Through a well-defined formula it is possible to calculate HMI (\%), as index of hair loss.

The Hair Breakage Index (HBI) calculates the hair breakage severity score in three sites of the selected bundle (from the scalp to the end extremity of the bundle) and it depends on the length of the bundle. The instrument automatically calculates the HBI. ${ }^{34}$

A hair score (1-3) was used to assess hair aspect as follow: $1=$ lacking of shininess; $2=$ a little bright; $3=$ shiny. ${ }^{14}$

Patients were instructed to not wash hair in the two days before clinic examination and to not apply cosmetics, gel, hair spray or any other topic lotion the 24 hours before.
Hair parameter clinical evaluation was assessed at baseline and after 12 weeks of treatment (follow-up).

\section{Statistical Analysis}

All data are presented as the means \pm SEM. A two-way Analysis of Variance (ANOVA) with Sidak's multiple comparisons test was used to analyze differences in skin, nails and hair parameters pre- and post-treatment. All statistical analyses were performed with GraphPad Prism 6 (GraphPad Software Inc., San Diego, CA, USA). p $<0.01$ was considered significant.

\section{RESULTS}

\section{Patients}

Seventeen patients $(50 \%)$ were affected by moderate VAS photoageing $(5.3+1.3 \mathrm{~cm})$ and 17 patients $(50 \%)$ were affected by severe VAS photoageing $(8.5+1.1 \mathrm{~cm})$ at baseline. General information about patients were resumed in Table 1, while Table 2 shows drugs daily swallowed. All these informations are expressed in terms of number $(\mathrm{N})$ of patient and the relative frequency ( $\%)$.

\section{Skin Parameters}

In the present study hydration significantly improved in all the selected area $(\mathrm{p}<0.01)$. Specifically, hydration increased of $6.9 \%$ in $\mathrm{F}$ (Figure 1a), of $6.1 \%$ in RMA (Figure 1b), of $6.5 \%$ in LMA (Figure 1c), of 6.8\% in RN (Figure 2a) and of $5.5 \%$ in $\mathrm{LN}$ (Figure 2b). Results of hydration at baseline and at follow-up are summarized in Table 3.

TEWL significantly improved in all the selected area $(p<0.001)$. Specifically, TEWL increased of $28.1 \%$ in F (Figure 3a), of $20.7 \%$ in RMA (Figure 3b), of $23.7 \%$ in LMA (Figure 3c), of $17.6 \%$ in RN (Figure 4a) and of $24.7 \%$ in LN (Figure 4b). Results of TEWL at baseline and at follow-up are summarized in Table 4.

Elasticity significantly improved in all the selected area $(\mathrm{p}<0.001)$. Specifically, elasticity increased of $17.1 \%$ in $\mathrm{F}$ (Figure 5a), of $27.1 \%$ in RMA (Figure $5 \mathrm{~b}$ ), of $23.2 \%$ in LMA (Figure 5c), of $22.9 \%$ in RN (Figure 6a) and of 26.1\% in LN (Figure 6b). Elasticity of FO (control) increased of $2 \%$. Results of elasticity at baseline and at follow-up are summarized in Table 5.

\section{Nails Parameters}

Nails hardness significantly improved in all the investigated hand fingers $(p<0.001)$. Specifically, nails hardness increased of $14.1 \%$ in IN (Figure $7 \mathrm{a}$ ), of $11.5 \%$ in ME (Figure 7b), of $13.5 \%$ in RI (Figure 7c). Results of nails hardness at baseline and at follow-up are summarized in Table 6. The nails score improved after 12 weeks of 


\begin{tabular}{|c|c|c|}
\hline Subjects & $\mathbf{N}$ & $\%$ \\
\hline Smokers & 11 & 32.4 \\
\hline$<5$ cigarettes & 1 & 9.1 \\
\hline $5-10$ cigarettes & 3 & 27.3 \\
\hline$>10$ cigarettes & 7 & 63.6 \\
\hline Non-smokers & 23 & 67.6 \\
\hline Menopause women & 23 & 74.2 \\
\hline Non-menopause women & 8 & 25.8 \\
\hline $\begin{array}{l}\text { Contraception or hormone } \\
\text { replacement therapy women }\end{array}$ & 4 & 12.9 \\
\hline $\begin{array}{l}\text { Non- contraception or } \\
\text { hormone replacement } \\
\text { therapy women }\end{array}$ & 27 & 87.1 \\
\hline Well-balanced diet & 27 & 79.4 \\
\hline Non-balanced diet & 7 & 20.6 \\
\hline Right-handed & 29 & 85.3 \\
\hline Left-handed & 5 & 14.7 \\
\hline \multicolumn{3}{|l|}{ Hair length } \\
\hline$<3 \mathrm{~cm}$ & 3 & 5.9 \\
\hline $3-11 \mathrm{~cm}$ & 15 & 44.1 \\
\hline$>11 \mathrm{~cm}$ & 7 & 50 \\
\hline
\end{tabular}

Table 2: Summary of daily drugs assumed by patients and relative number of patients $(\mathrm{N})$ and frequency $(\%)$ of consumption

\begin{tabular}{lcc} 
Drugs & N & $\%$ \\
\hline Statins & 2 & 4.9 \\
Analgesics & 2 & 4.9 \\
Anti-reflux & 3 & 7.3 \\
Antihistaminic & 1 & 2.5 \\
Thyroid drugs & 6 & 14.6 \\
Antihypertensive & 5 & 12.2 \\
Supplements & 6 & 14.6 \\
No drug therapy & 16 & 39.0 \\
Total drugs & 41 & 100 \\
\hline
\end{tabular}

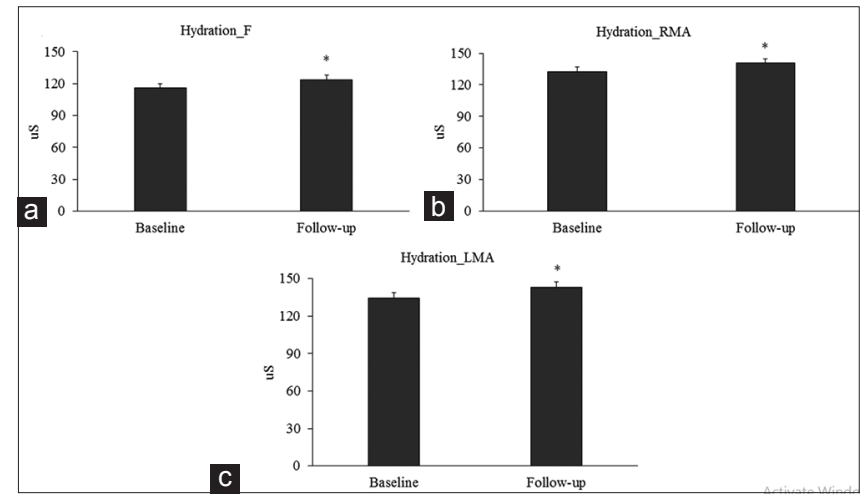

Figure 1: Skin hydration of forehead (a), right malar area (b), and left malar area (c) significantly increased in patients affected by age-related morphological anatomic changes after 12 weeks of treatment (follow-up). * $\mathrm{p}<0.01$

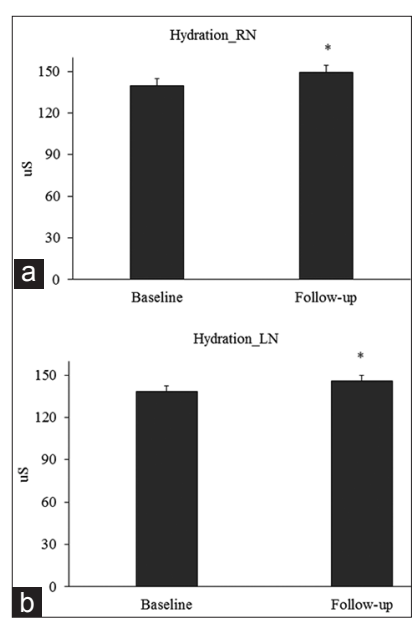

Figure 2: Skin hydration of right lateral - medial area of the neck (a) and left lateral-medial area of the neck (b) significantly increased in patients affected by age-related morphological anatomic changes after 12 weeks of treatment (follow-up). * $p<0.01$

\begin{tabular}{|c|c|c|c|c|c|c|c|c|c|c|}
\hline \multirow[t]{2}{*}{ Area } & \multicolumn{2}{|c|}{ Mean } & \multicolumn{2}{|c|}{ Median } & \multicolumn{2}{|c|}{ SD } & \multicolumn{2}{|c|}{ Min } & \multicolumn{2}{|c|}{ Max } \\
\hline & Before & Follow-up & Before & Follow-up & Before & Follow-up & Before & Follow-up & Before & Follow-up \\
\hline $\mathrm{F}$ & 115.93 & 123.92 & 108.7 & 113.3 & 36.3 & 36.8 & 60 & 81 & 237 & 256 \\
\hline RMA & 132.75 & 140.80 & 132 & 140 & 35.5 & 39.4 & 77 & 80 & 196 & 213.6 \\
\hline LMA & 134.44 & 143.24 & 134 & 143 & 39.6 & 41.8 & 76 & 80 & 226 & 234 \\
\hline RN & 139.87 & 149.42 & 134 & 145.5 & 42.4 & 45.3 & 60 & 72 & 219 & 234.5 \\
\hline LN & 138.36 & 145.99 & 135.5 & 150.9 & 39.0 & 40.7 & 65 & 72 & 212 & 235.7 \\
\hline
\end{tabular}

Table 4: Mean value, median, standard deviation (SD), minimum (min) and maximum (max) value of TEWL in the selected area at baseline and after 12 weeks of treatment (follow-up). Data are expressed in $\mathrm{g} / \mathrm{m} 2 / \mathrm{h}$

\begin{tabular}{|c|c|c|c|c|c|c|c|c|c|c|}
\hline \multirow[t]{2}{*}{ Area } & \multicolumn{2}{|c|}{ Mean } & \multicolumn{2}{|c|}{ Median } & \multicolumn{2}{|c|}{ SD } & \multicolumn{2}{|c|}{ Min } & \multicolumn{2}{|c|}{ Max } \\
\hline & Before & Follow-up & Before & Follow-up & Before & Follow-up & Before & Follow-up & Before & Follow-up \\
\hline $\mathrm{F}$ & 4.49 & 5.75 & 4.5 & 5.1 & 2.5 & 2.5 & 1 & 2.2 & 9.7 & 11.5 \\
\hline RMA & 5.56 & 6.71 & 4.4 & 5.75 & 3.4 & 3.5 & 1 & 1.8 & 11.1 & 15.6 \\
\hline LMA & 5.0 & 6.19 & 4.7 & 5.6 & 3 & 3.1 & 1.4 & 1.8 & 10.3 & 12.3 \\
\hline $\mathrm{RN}$ & 6.11 & 7.19 & 5.75 & 6.75 & 3.8 & 4.0 & 1.1 & 1.6 & 13.5 & 14.5 \\
\hline LN & 5.36 & 6.69 & 5.65 & 6.55 & 3.0 & 3.4 & 1.3 & 1.6 & 15.7 & 16.8 \\
\hline
\end{tabular}




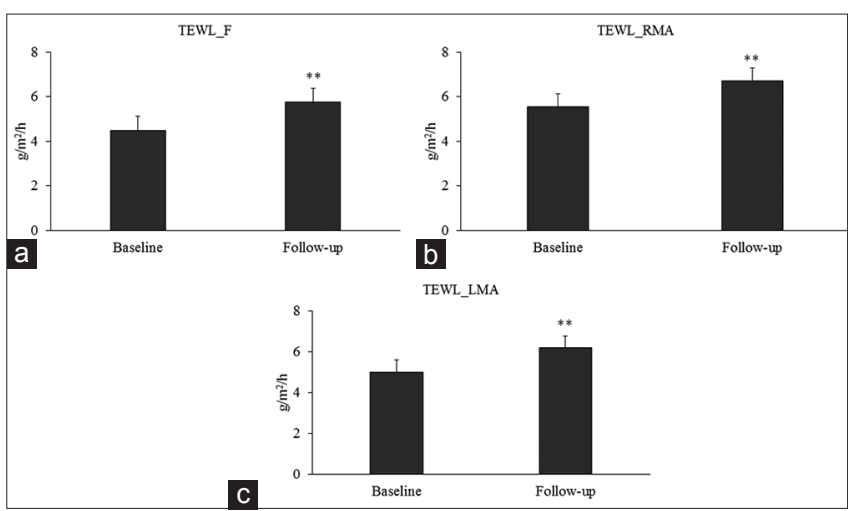

Figure 3: Skin TEWL of forehead (a), right malar area (b), and left malar area (c) significantly increased in patients affected by age-related morphological anatomic changes after 12 weeks of treatment (follow-up). ${ }^{* *} p<0.001$

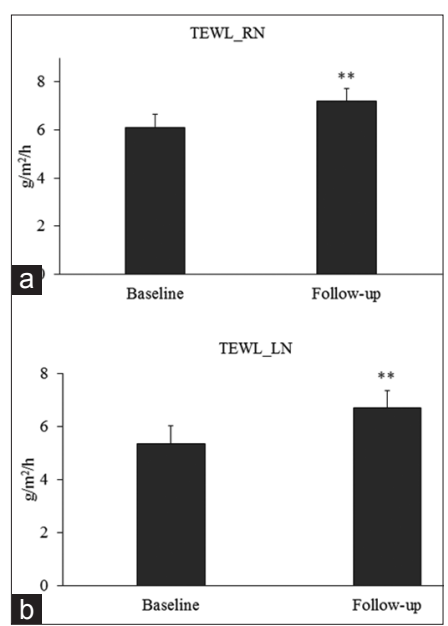

Figure 4: Skin TEWL of right lateral - medial area of the neck (a) and left lateral-medial area of the neck (b) significantly increased in patients affected by age-related morphological anatomic changes after 12 weeks of treatment (follow-up). ${ }^{* *} p<0.001$

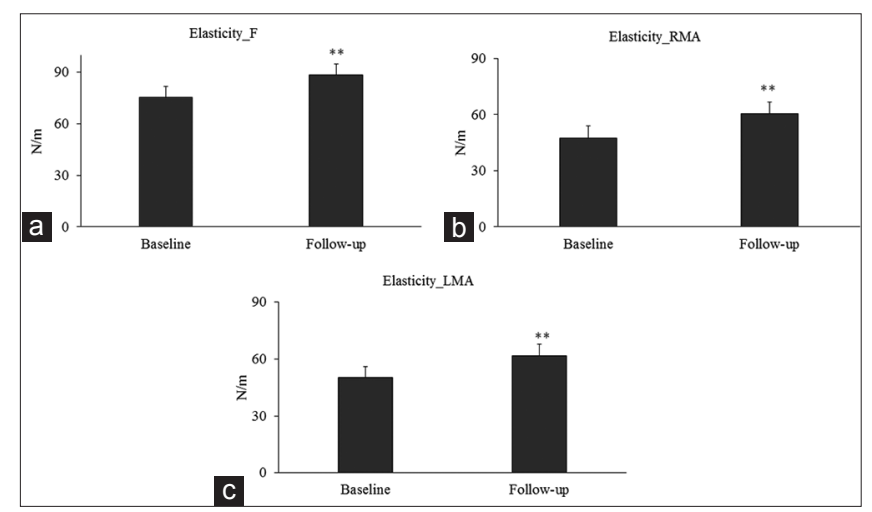

Figure 5: Skin elasticity of forehead (a), right malar area (b), and left malar area (c) significantly increased in patients affected by age-related morphological anatomic changes after 12 weeks of treatment (follow-up). ${ }^{* *} p<0.001$

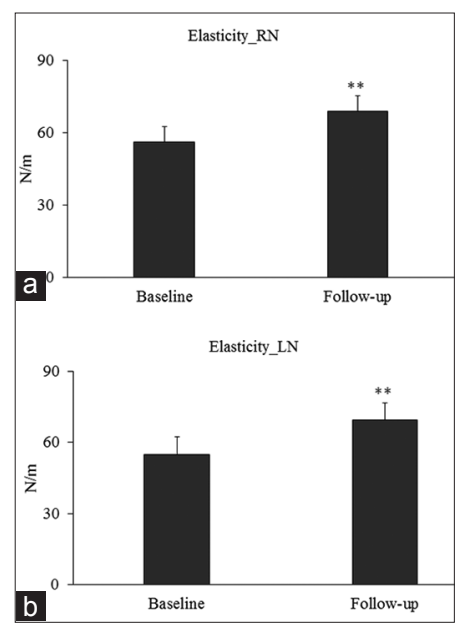

Figure 6: Skin elasticity of right lateral - medial area of the neck (a) and left lateral-medial area of the neck (b) significantly increased in patients affected by age-related morphological anatomic changes after 12 weeks of treatment (follow-up). ${ }^{* *} p<0.001$

\begin{tabular}{|c|c|c|c|c|c|c|c|c|c|c|}
\hline \multirow[t]{2}{*}{ Area } & \multicolumn{2}{|c|}{ Mean } & \multicolumn{2}{|c|}{ Median } & \multicolumn{2}{|c|}{ SD } & \multicolumn{2}{|c|}{ Min } & \multicolumn{2}{|c|}{ Max } \\
\hline & Before & Follow-up & Before & Follow-up & Before & Follow-up & Before & Follow-up & Before & Follow-up \\
\hline $\mathrm{F}$ & 75.29 & 88.21 & 75.2 & 87 & 32.8 & 35.2 & 23 & 32 & 133 & 156 \\
\hline RMA & 47.47 & 60.35 & 41.5 & 59 & 18.5 & 18.5 & 25 & 31 & 89 & 99 \\
\hline LMA & 50.21 & 61.85 & 44 & 56 & 19.6 & 17.7 & 20 & 34 & 91 & 97 \\
\hline RN & 56.03 & 68.98 & 50 & 67.5 & 19.6 & 21.2 & 24 & 32 & 93 & 126 \\
\hline LN & 55.06 & 69.41 & 48 & 66.5 & 21.3 & 21.4 & 20 & 30 & 99 & 103 \\
\hline $\begin{array}{l}\text { FO } \\
\text { (control) }\end{array}$ & 61.59 & 62.82 & 66 & 66 & 23.6 & 23.3 & 23 & 23 & 98 & 99 \\
\hline
\end{tabular}

Table 6: Mean value, median, standard deviation (SD), minimum (min) and maximum (max) value of nails hardness in all the investigated hand fingers at baseline and after 12 weeks of treatment (follow-up). Data are expressed in Shore A

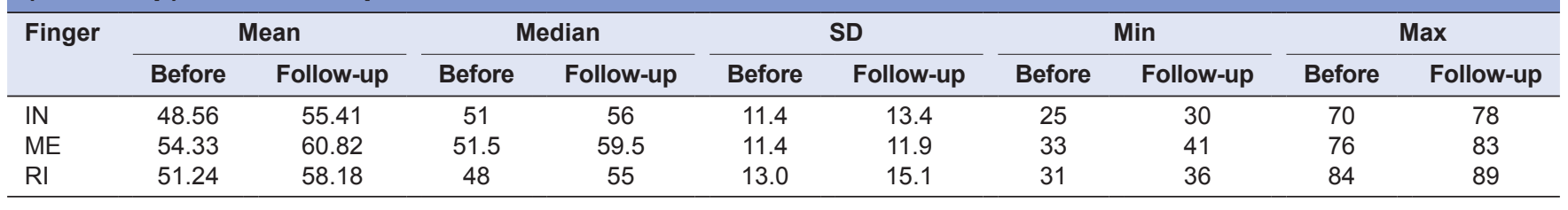

Asian Journal of Medical Sciences | May-Jun 2017 | Vol 8 | Issue 3 
treatment (Figure 8). At baseline 3 patients $(8.8 \%)$ had nails score 1, 5 patients $(14.7 \%)$ had nails score 2,18 patients $(52.9 \%)$ had nails score 3 and 8 patients $(23.5 \%)$ had nails score 4 . At follow-up any patient $(0 \%)$ had score 1,1 patient $(2.9 \%)$ had nails score 2,13 patients $(38.2 \%)$ had nails score 3 and 20 patients had nails score 4 (58.8\%).

\section{Hair Parameter}

HMI and HBI significantly improved $(\mathrm{p}<0.0001)$. Specifically, HMI that is an index of $\%$ hair loss decreased of $84.9 \%$ (Figure 9a), and HBI that is an index

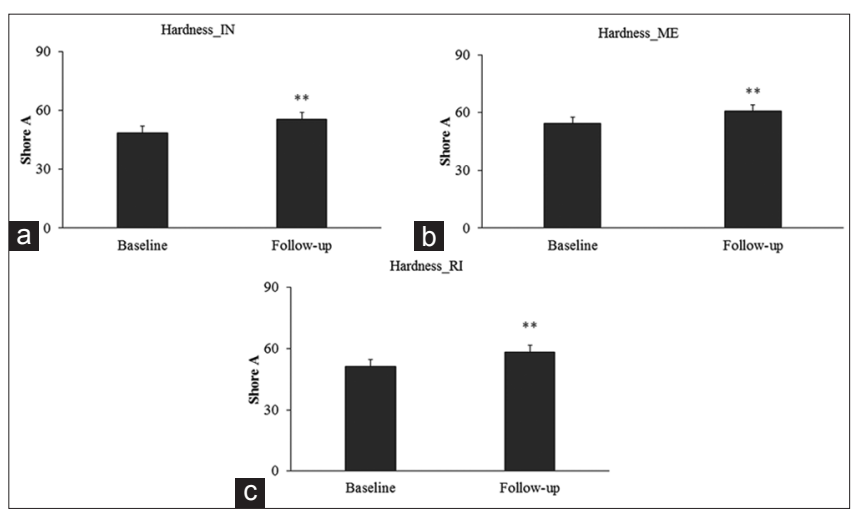

Figure 7: Nails hardness of index finger (a), middle finger (b) and ring finger (c) significantly increased in patients affected by age-related morphological anatomic changes after 12 weeks of treatment (follow-up). ${ }^{* \star} p<0.001$

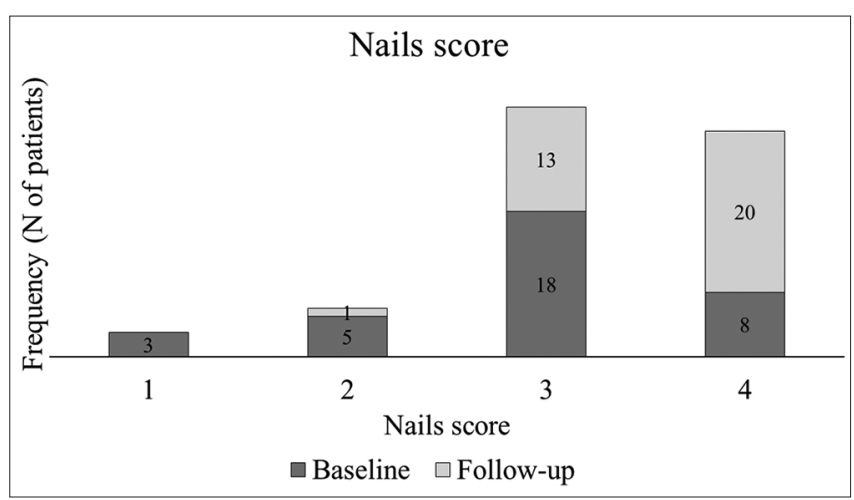

Figure 8: Frequency (Number of patients) of the nails score (1-4) at baseline and after 12 weeks of treatment (follow-up). Nails score improved after 12 weeks of treatment $(n=34)$ of $\%$ hair breakage decreased of $64.1 \%$ (Figure 9b). Results of HMI and HBI at baseline and at follow-up are summarized in Table 7 . The hair score improved after 12 weeks of treatment (Figure 10). At baseline one patient $(2.9 \%)$ had hair score 1,15 patients $(44.1 \%)$ had hair score 2 , and 18 patients $(52.9 \%)$ had hair score 3. At follow-up any patient $(0 \%)$ had hair score 1 , one patient $(2.9 \%)$ had hair score 2 , and 33 patients $(97.1 \%)$ had hair score 3.

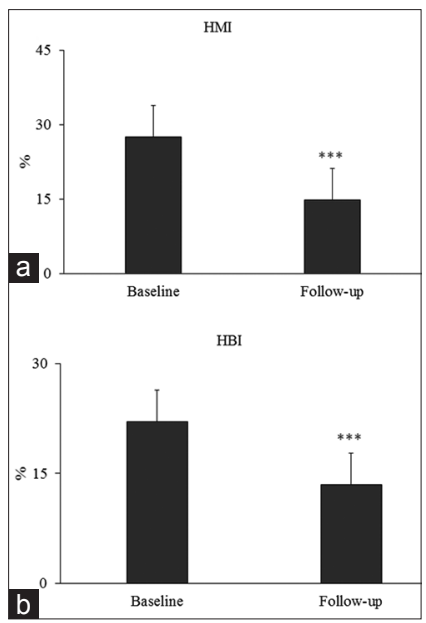

Figure 9: Hair Mass Index (a) and Hair Breakage Index (b) significantly decreased in patients affected by age-related morphological anatomic changes after 12 weeks of treatment (follow-up). ${ }^{* \star *} p<0.0001$

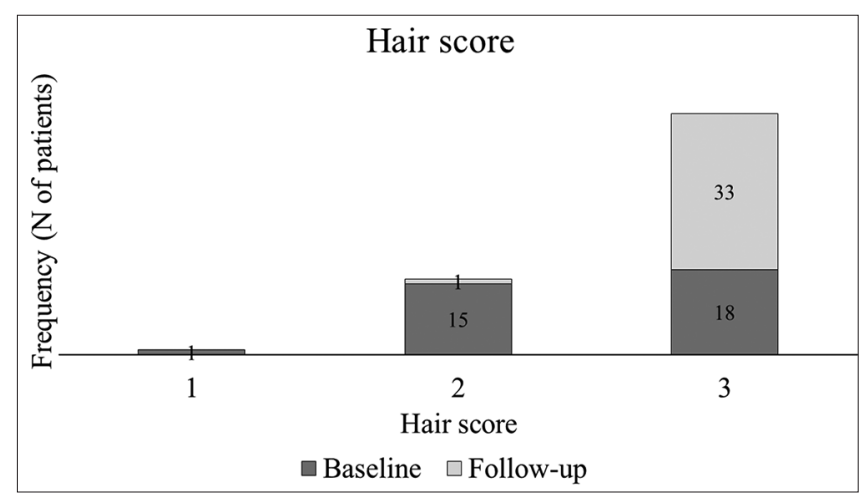

Figure 10: Frequency (Number of patients) of the hair score (1-3) at baseline and after 12 weeks of treatment (follow-up). Hair score improved after 12 weeks of treatment $(n=34)$

\begin{tabular}{|c|c|c|c|c|c|c|c|c|c|c|}
\hline \multirow[t]{2}{*}{ Area } & \multicolumn{2}{|c|}{ Mean } & \multicolumn{2}{|c|}{ Median } & \multicolumn{2}{|c|}{ SD } & \multicolumn{2}{|c|}{ Min } & \multicolumn{2}{|c|}{ Max } \\
\hline & Before & Follow-up & Before & Follow-up & Before & Follow-up & Before & Follow-up & Before & Follow-up \\
\hline $\begin{array}{l}\text { Control } \\
\text { HMI }\end{array}$ & 0.0418 & 0.0418 & 0.0447 & 0.0447 & 0.013 & 0.013 & 0.00187 & 0.00187 & 0.0587 & 0.0587 \\
\hline $\begin{array}{l}\text { Affected } \\
\mathrm{HMI}\end{array}$ & 0.0332 & 0.0486 & 0.0347 & 0.0402 & 0.009 & 0.008 & 0.0176 & 0.0186 & 0.047 & 0.431 \\
\hline HMI (\%) & 27.51 & 14.88 & 22.35 & 12.15 & 21.1 & 12.2 & 7.6 & 0.0 & 93.7 & 42.27 \\
\hline HBI (\%) & 22.03 & 13.43 & 20.0 & 11.6 & 15.0 & 10.4 & 1.2 & 0.7 & 52.04 & 43.9 \\
\hline
\end{tabular}




\section{DISCUSSION}

In our study, the administration of a dietary supplement containing Pycnogenol ${ }^{\circledR}$, low-molecular-weight HA, collagen, glucosamine sulfate, chondroitin sulfate and coenzyme Q10 significantly increased skin face hydration, TEWL elasticity, nails hardness and significantly reduced hair loss and hair breakage in patients affected by age-related dermatological changes, after 12-weeks treatment period. The changes of nails and hair parameters fit with nails and hair scores improvement, especially on nails whiteness and rigidity of nails and shining of hair. Modulation of these parameters by the dietary supplementation may be useful to counteract some of the age-related dermatological changes, accordingly with previous findings, where the same supplement significantly improved VAS photoaging score, facial sebum, hydration and tonicity from the second week of treatment. ${ }^{24}$

Collagen was added into the formula since experimental evidences from murine models has shown that: if adequately orally administered it prevents UV radiationinduced skin damage. ${ }^{35,36}$ In a study by Proksch et al., oral supplementation of collagen hydrolysate composed of specific collagen peptides $(2.5 \mathrm{~g} / \mathrm{d}$ or $5.0 \mathrm{~g} / \mathrm{d}$ for 8 weeks) increased skin elasticity in middle-aged women after 4 weeks of supplementation. ${ }^{37}$ Previous studies confirmed that collagen hydrolysate is degraded to small peptides by the gastrointestinal tract, that appear later in the blood stream and reach the skin in 8 up to 96 hours. ${ }^{38}$ These peptides are chemotactic for dermal fibroblasts, ${ }^{39}$ and promote the migration and growth of mouse skin fibroblasts. ${ }^{40}$ In addition, collagen is a physiological constituent of nails structure, contributing with keratin to its strength. ${ }^{41}$ However, no studies either in experimental models or in clinical trials has been published about nails and hair parameters pre-post oral collagen supplementation.

HA was also added to the dietary formulation in a cosmeceutical perspective because of its hydrophilic properties, dermal elasticity, metabolite and nutrient spreading in the interstitium. ${ }^{42}$

As to the mucopolysaccharides oral administration, the rationale is to compensate the reduced amount of HA found in innate ageing, ${ }^{43}$ such as wrinkling and altered elasticity, ${ }^{43}$ being the CD44, HA-high affinity cell surface receptor largely expressed in hair follicles in murine models. ${ }^{44}$

Chondroitin sulfate is intrinsic factor of the biosynthesis of collagen and elastic fibres formation. ${ }^{45}$ Previous studies showed that if integrated with other natural compounds, aminoacids, minerals, and various antioxidant compounds, it improves cutaneous aging-induced wrinkles ${ }^{36}$ and stimulates hair follicles stem cells. ${ }^{46}$ In fact, chondroitin sulfate binds and modulates the functions of a large number of biomolecules involved in hair follicle differentiation or proliferation, ${ }^{47}$ including fibroblast growth factors (FGFs), ${ }^{48}$ vascular endothelial growth factor (VEGF), ${ }^{49}$ sonic hedgehog, ${ }^{50}$ bone morphogenetic proteins (BMPs) ${ }^{51} \mathrm{Wnt}^{50}$ and hepatocyte growth factor (HGF). ${ }^{51}$ Chondroitin sulfate is also a physiological component with extracellular matrix (ECM) glycoproteins, such as laminin of the nails framework, ${ }^{52}$ supporting the integrity of the lamina. In addition, glucosamine sulfate, inhibited UVBinduced collagen-lytic matrix metalloproteinases (MMP) production through different pathways in immortalized human skin fibroblasts. ${ }^{53}$

Last, but not least, the antioxidants included in the dietary supplement have the property of counteracting cellular oxidative stress in different conditions. ${ }^{54,55}$ Topical Pycnogenol ${ }^{\circledR}$ and oral coenzyme Q10 are quite effective in preventing oxidative stress of skin ageing, ${ }^{56}$ and Pycnogenol ${ }^{\circledR}$ alone improved skin elasticity and hydration in women presenting dry skin, ${ }^{57}$ reduced the hair bulbs atrophy, increased the vascular network and reduced the necrotic areas in rat incisional wound model..$^{58}$ Quantitatively a cream containing 2\% Pycnogenol $^{\circledR}$ inhibited the skin lipid peroxidation by a $35 \%$, by a $35 \%$ decreased malondialdehyde (MDA) and $46.8 \%$ increase in superoxide dismutase (SOD) activity, after 9 days.

Coenzyme Q10 increased fibroblasts proliferation, the expression of type IV collagen and reduced UV radiation-induced MMP-1 level in embryonic and adult cells. ${ }^{59}$ In addition, treatment with coenzyme Q10 increased elastin gene expression in cultured fibroblasts and decreased UV radiation-induced interleukin-1 alpha (IL-1 a) production in aneuploid immortal keratinocyte $(\mathrm{HaCaT})$ cells $^{59}{ }^{59} \mathrm{~A}$ randomized placebo controlled study showed that coenzyme Q10 oral supplement reduced dry skin in patients affected by acne after 6 months of treatment. ${ }^{60}$ Finally, a 21 days of dietary supplementation containing coenzyme Q10 (0.31g/100 g) significantly reduced stressed-noise induced hair loss in a SpragueDawley rat model, specifically, preventing oxidative stress apoptosis. ${ }^{61}$

In conclusion, a 12 -weeks oral administration of Pycnogenol $^{\circledR}$, HA, collagen, glucosamine sulfate, chondroitin sulfate and coenzyme Q10, displayed a synergistic ECM, modulating effect with clinical benefit on skin barrier, nails and hair impairment. Future studies will also be addressed to the long-term effects of this formulation in specific skin and adnexa syndromes. 


\section{REFERENCES}

1. Hardy $\mathrm{MH}$. The secret life of the hair follicle. Trends Genet 1992;8:55-61.

2. Paus R and Foitzik K. In search of the "hair cycle clock": a guided tour. Differentiation. 2004;72:489-511.

3. Langbein $L$ and Schweizer J. Keratins of the human hair follicle. Int Rev Cytol 2005;243:1-78.

4. Daniel CR, Sams WM and Scher RK. Nails in systemic disease. Dermatol Clin 1985;3:465-483.

5. Cummings DM and Uttech KM. Antibiotics for common infections in the elderly. Prim Care 1990;17:883-903.

6. Sjerobabski-Masnec I and Situm M. Skin aging. Acta Clin Croat 2010;49:515-518.

7. Gavazzi G and Krause $\mathrm{KH}$. Ageing and infection. Lancet Infect Dis 2002;2:659-666.

8. Mestre-Deharo C and Sayag J. Histologic signs of cutaneous aging. Rev Fr Gynecol Obstet 1991;86:425-432.

9. Laube S. Skin infections and ageing. Ageing Res Rev 2004;3:69-89.

10. Thurstan SAand Gibbs NK, Langton AK. Chemical consequences of cutaneous photoageing. Chem Cent J 2012;6:34.

11. D'Orazio J, Jarrett $S$, Amaro-Ortiz A and Scott T. UV radiation and the skin. Int J Mol Sci 2013;14:12222-12248.

12. Trueb RM. Effect of ultraviolet radiation, smoking and nutrition on hair. Curr Probl Dermatol 2015;47:107-120.

13. Klonizakis M, Alkhatib A and Middleton G. Long-term effects of an exercise and Mediterranean diet intervention in the vascular function of an older, healthy population. Microvasc Res 2014 Sep;95:103-107.

14. Beer $\mathrm{C}$, Wood $\mathrm{S}$ and Veghte $\mathrm{RH}$. A clinical trial to investigate the effect of Cynatine HNS on hair and nail parameters. Scientific World Journal 2014;2014:641723.

15. Dickinson A, Shao A, Boyon $\mathrm{N}$ and Franco JC. Use of dietary supplements by cardiologists, dermatologists and orthopedists: report of a survey. Nutr J 2011;10:20.

16. Jacquet $A$, Coolen $V$ and Vandermander J. Effect of dietary supplementation with INVERSION Femme on slimming, hair loss, and skin and nail parameters in women. Adv Ther 2007;24:1154-1171.

17. Alhaj $\mathrm{E}$, Alhaj $\mathrm{N}$ and Alhaj NE. Diffuse alopecia in a child due to dietary zinc deficiency. Skin med 2007;6:199-200.

18. Arroyo JF and Cohen ML. Improvement of yellow nail syndrome with oral zinc supplementation. Clin Exp Dermatol 1993;18:62-64.

19. Rayman MP, Searle E and Kelly L. Effect of selenium on markers of risk of pre-eclampsia in UK pregnant women: a randomised, controlled pilot trial. Br J Nutr 2014;112:99-111.

20. Alexander J. Selenium. Novartis Found Symp 2007;282:143-9.

21. Budde J, Tronnier H, Rahlfs VW and Frei-Kleiner S. Systemic therapy of diffuse effluvium and hair structure damage. Hautarzt 1993;44:380-384.

22. Boccaletti V, Zendri E, and Giordano G. Familial Uncombable Hair Syndrome: Ultrastructural Hair Study and Response to Biotin. Pediatr Dermatol 2007;24:E14-E16.

23. Petri $H$, Pierchalla $P$ and Tronnier $H$. The efficacy of drug therapy in structural lesions of the hair and in diffuse effluvium-comparative double blind study. Schweiz Rundsch Med Prax 1990;79:1457-1462.

24. Di Cerbo A, Laurino C, Palmieri B and lannitti T. A dietary supplement improves facial photoaging and skin sebum, hydration and tonicity modulating serum fibronectin, neutrophil elastase 2, hyaluronic acid and carbonylated proteins. J Photochem Photobiol B 2015;144:94-103.

25. Marks $R$ and Shahrad $P$. Skin changes at the time of the climacteric. Clin Obstet Gynaecol 1977;207-226.

26. Laurino C, Palmieri B and Coacci A. Efficacy, Safety, and Tolerance of a New Injection Technique for High- and LowMolecular-Weight Hyaluronic Acid Hybrid Complexes. Eplasty 2015;15:e46.

27. Boireau-Adamezyk E, Baillet-Guffroy A and Stamatas GN. Agedependent changes in stratum corneum barrier function. Skin Res Technol 2014;20:409-415.

28. Elias PM. Stratum corneum defensive functions: an integrated view. J Invest Dermatol 2005 Aug;125:183-200.

29. livarinen JT, Korhonen RK and Jurvelin JS. Experimental and numerical analysis of soft tissue stiffness measurement using manual indentation device--significance of indentation geometry and soft tissue thickness. Skin Res Technol 2014;20:347-354.

30. Robson JR and el-Tahawi HD. Hardness of human nail as an index of nutritional status: a preliminary communication. $\mathrm{Br} \mathrm{J}$ Nutr 1971;26:233-236.

31. Robson JR. Hardness of finger nails in well-nourished and malnourished populations. Br J Nutr 1974;32:389-394.

32. van de Kerkhof PC, Pasch MC, Scher RK and Kerscher M. Brittle nail syndrome: a pathogenesis-based approach with a proposed grading system. J Am Acad Dermatol 2005;53:644-651.

33. Cohen B. The cross-section trichometer: a new device for measuring hair quantity, hair loss, and hair growth. Dermatol Surg 2008;34:900-910.

34. Hendriks MA, Geerts PA and Dercksen. Evaluation of Cohen's cross-section trichometer for measuring hair quantity. Dermatol Surg 2012;38:631-634.

35. Zhuang $\mathrm{Y}$, Hou $\mathrm{H}$, and Zhao X. Effects of collagen and collagen hydrolysate from jellyfish (Rhopilema esculentum) on mice skin photoaging induced by UV irradiation. J Food Sci 2009;74:H183-H188.

36. Murad $\mathrm{H}$ and Tabibian MP. The effect of an oral supplement containing glucosamine, amino acids, minerals, and antioxidants on cutaneous aging: a preliminary study. J Dermatolog Treat 2001;12:47-51.

37. Proksch E, Segger D and Degwert J. Oral supplementation of specific collagen peptides has beneficial effects on human skin physiology: a double-blind, placebo-controlled study. Skin Pharmacol Physiol 2014;27:47-55.

38. Oesser S, Adam M, Babel W and Seifert J. Oral administration of (14)C labeled gelatin hydrolysate leads to an accumulation of radioactivity in cartilage of mice (C57/BL). J Nutr 1999;129:1891-1895.

39. Postlethwaite AE, Seyer JM and Kang AH. Chemotactic attraction of human fibroblasts to type I, II, and III collagens and collagenderived peptides. Proc Natl Acad Sci U S A 1978;75:871-875.

40. Shigemura $\mathrm{Y}$, Iwai $\mathrm{K}$ and Morimatsu F. Effect of Prolylhydroxyproline (Pro-Hyp), a food-derived collagen peptide in human blood, on growth of fibroblasts from mouse skin. J Agric Food Chem 2009;57:444-449.

41. Campeau E, Watkins $D$ and Rouleau GA. Linkage analysis of the nail-patella syndrome. Am J Hum Genet 1995;56:243-247.

42. Dai G, Freudenberger $T$ and Zipper $P$. Chronic ultraviolet B irradiation causes loss of hyaluronic acid from mouse dermis because of down-regulation of hyaluronic acid synthases. Am J Pathol 2007;171:1451-1461.

43. Ghersetich I, Lotti $\mathrm{T}$ and Campanile G. Hyaluronic acid in cutaneous intrinsic aging. Int J Dermatol 1994;33:119-122.

44. Kaya G, Augsburger E, Chavaz P and Saurat JH. CD44 and 
hyaluronate expression in follicular mucinosis. J Cutan Pathol 2006;33:227-230.

45. Kielty CM, Whittaker SP and Shuttleworth CA. Fibrillin: evidence that chondroitin sulphate proteoglycans are components of microfibrils and associate with newly synthesised monomers. FEBS Lett 1996;386:169-173.

46. Quan R, Zheng $X$ and $X u$ S. Gelatin-chondroitin-6-sulfatehyaluronic acid scaffold seeded with vascular endothelial growth factor 165 modified hair follicle stem cells as a three-dimensional skin substitute. Stem Cell Res Ther 2014;5:118.

47. Malgouries S, Thibaut $S$ and Bernard BA. Proteoglycan expression patterns in human hair follicle. $\mathrm{Br} J$ Dermatol 2008;158:234-242.

48. Pellegrini L. Role of heparan sulfate in fibroblast growth factor signalling: a structural view. Curr Opin Struct Biol 2001;11:629-634.

49. Cohen T, Gitay-Goren H, Sharon R, Shibuya M, Halaban R, Levi BZ, et al. VEGF121, a vascular endothelial growth factor (VEGF) isoform lacking heparin binding ability, requires cell-surface heparan sulfates for efficient binding to the VEGF receptors of human melanoma cells. J Biol Chem 1995;270:11322-11326

50. Bornemann DJ, Duncan JE and Staatz W. Abrogation of heparan sulfate synthesis in Drosophila disrupts the Wingless, Hedgehog and Decapentaplegic signaling pathways. Development 2004;131:1927-1938.

51. Takada T, Katagiri T and Ifuku M. Sulfated polysaccharides enhance the biological activities of bone morphogenetic proteins. J Biol Chem 2003;278:43229-43235.

52. Sinclair RD, Wojnarowska F, Leigh $I M$ and Dawber RP The basement membrane zone of the nail. $\mathrm{Br} J$ Dermatol
1994;131:499-505.

53. Hwang YP, Kim HG and Han EH. N-Acetylglucosamine suppress collagenases activation in ultraviolet B-irradiated human dermal fibroblasts: Involvement of calcium ions and mitogen-activated protein kinases. J Dermatol Sci 2011;63:93-103.

54. Iannitti $T$ and Palmieri B. Antioxidant therapy effectiveness: an up to date. Eur Rev Med Pharmacol Sci 2009;13:245-278.

55. Iannitti $T$, Rottigni $\vee$ and Palmieri $B$. Role of free radicals and antioxidant defences in oral cavity-related pathologies. J Oral Pathol Med 2012;41:649-661.

56. Berson DS. Natural antioxidants. J Drugs Dermatol 2008;7:s7-s12.

57. Marini A, Grether-Beck S and Jaenicke T. Pycnogenol(R) effects on skin elasticity and hydration coincide with increased gene expressions of collagen type I and hyaluronic acid synthase in women. Skin Pharmacol Physiol 2012;25:86-92.

58. Cetin EO, Yesil-Celiktas $O$ and Cavusoglu T. Incision wound healing activity of pine bark extract containing topical formulations: a study with histopathological and biochemical analyses in albino rats. Pharmazie 2013;68:75-80.

59. Zhang M, Dang L and Guo F. Coenzyme Q(10) enhances dermal elastin expression, inhibits IL-1alpha production and melanin synthesis in vitro. Int J Cosmet Sci 2012;34:273-279.

60. Fabbrocini G, Cameli N and Lorenzi S. A dietary supplement to reduce side effects of oral isotretinoin therapy in acne patients. G Ital Dermatol Venereol 2014;149:441-445.

61. Cascella V, Giordano P and Hatzopoulos S. A new oral otoprotective agent. Part 1: Electrophysiology data from protection against noise-induced hearing loss. Med Sci Monit 2012;18:BR1-BR8.

\section{Authors Contribution:}

CL- Concept and design of the study, manuscript preparation, statistically analyzed and interpreted, critical revision of the manuscript; reviewed the literature helped in preparing first draft of manuscript, collected data; BP-Concept and design of the study, manuscript preparation, statistically analyzed and interpreted, critical revision of the manuscript; AC- reviewed the literature, helped in preparing first draft of manuscript, collected data; MV- reviewed the literature, helped in preparing first draft of manuscript.

Orcid ID:

Dr Carmen Laurino: (1) http://orcid.org/0000-0002-3020-2338

Prof. Beniamino Palmieri: (1) http://orcid.org/0000-0002-0871-138X

Dr Alessandro Coacci: (i) http://orcid.org/0000-0003-1167-1486

Dr Maria Vadalà: (i) http://orcid.org/0000-0001-7873-5072

Source of Support: None, Conflict of Interest: The authors hereby certify that all work contained in this article is original. The authors claim full responsibility for the contents of the article. The authors contributed equally to this work. This article was not supported by grants. The authors certify that there is no conflict of interest with any financial organization regarding the material discussed in the manuscript. 